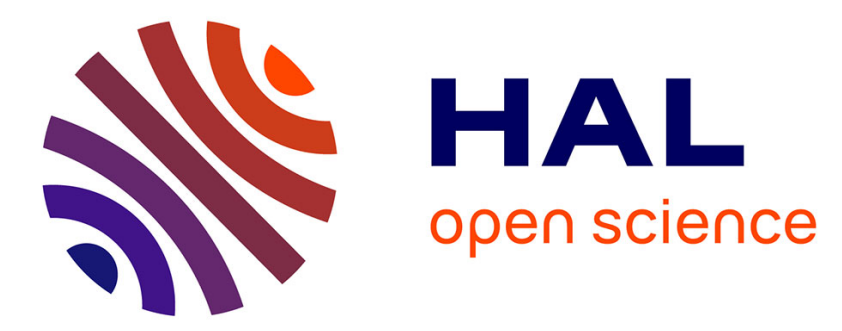

\title{
Évolution de la réponse photosynthétique des feuilles et efficience théorique de la photosynthèse brute d'une culture de canne à sucre (Saccharum officinarum L.)
}

Claude Varlet-Grancher, Raymond Bonhomme, Michel Chartier, Philippe Artis

\section{To cite this version:}

Claude Varlet-Grancher, Raymond Bonhomme, Michel Chartier, Philippe Artis. Évolution de la réponse photosynthétique des feuilles et efficience théorique de la photosynthèse brute d'une culture de canne à sucre (Saccharum officinarum L.). Agronomie, 1981, 1 (6), pp.473-481. hal-00884283

\section{HAL Id: hal-00884283 https://hal.science/hal-00884283}

Submitted on 1 Jan 1981

HAL is a multi-disciplinary open access archive for the deposit and dissemination of scientific research documents, whether they are published or not. The documents may come from teaching and research institutions in France or abroad, or from public or private research centers.
L'archive ouverte pluridisciplinaire HAL, est destinée au dépôt et à la diffusion de documents scientifiques de niveau recherche, publiés ou non, émanant des établissements d'enseignement et de recherche français ou étrangers, des laboratoires publics ou privés. 


\title{
Évolution de la réponse photosynthétique des feuil- les et efficience théorique de la photosynthèse brute d'une culture de canne à sucre (Saccharum officina-
} rum L.)

\author{
Claude VARLET-GRANCHER $\left({ }^{*}\right)$, Raymond BONHOMME $\left({ }^{*}\right)$, Michel CHARTIER $\left({ }^{* *}\right)$ \& Philippe \\ ARTIS (***) \\ (*) I.N.R.A., Laboratoire de Bioclimatologie, Domaine Brunehaut F 80200 Péronne. \\ (**) I.N.R.A., Station de Bioclimatologie, route de St-Cyr, F 78000 Versailles. \\ ${ }_{(* * *)}^{* * N}$ I.R.A., Station de Bioclimatologie, Centre de recherches agronomiques des Antilles et de la Guyane, \\ GP 97170 Petit-Bourg.
}

RÉSUMÉ

Photosynthèse feuille, Plateau de saturation, Âge,

Efficience,

Photosynthèse,

Production culture,

Canne à sucre.

\begin{abstract}
La photosynthèse brute d'une culture de canne à sucre (Saccharum officinarum L.) est caractérisée par son efficience journalière $\varepsilon_{\mathrm{PB}}$ définie comme le rapport entre l'intensité de la photosynthèse brute PB et le rayonnement utile à la photosynthèse absorbée par la végétation.

La valeur journalière de $\mathrm{PB}$ est estimée à partir des réponses photosynthétiques des feuilles (fig. 1) de leur éclairement et de la structure de la végétation.

Les évolutions de l'assimilation nette maximale de l'unité de surface de feuilles $\mathrm{N}_{\mathrm{m}}$ pour différentes feuilles au cours du développement de la culture sont montrées à la figure 2 alors que la figure 3 donne l'effet du vieillissement du couvert végétal sur $\mathrm{N}_{\mathrm{m}}$ pour des feuilles de rang 2 ou 3 de différentes variétés.

L'influence théorique des principaux facteurs: rayonnement incident, indice foliaire, caractéristiques photosynthétiques des feuilles..., sur l'efficience de la photosynthèse brute de la culture est montrée par la figure 4 .

L'évolution de $\varepsilon_{\mathrm{PB}}$ obtenue à partir des valeurs de ces différents paramètres mesurées sur une culture à plusieurs stades de sa croissance est donnée sur la figure 5 . Les pertes par respiration $\left(\varepsilon_{\mathrm{RD}}\right)$ ont été estimées pour calculer une efficience de la photosynthèse nette $\left(\varepsilon_{\mathrm{b}}^{\prime}=\varepsilon_{\mathrm{PB}}=\varepsilon_{\mathrm{RD}}\right)$ et comparées au rendement de la conversion du rayonnement solaire en matière sèche $\left(\varepsilon_{\mathrm{b}}\right)$.
\end{abstract}

\section{SUMMARY}

Leaf photosynthesis, Saturation,

Age,

Photosynthetic efficiency, Crop production, Sugarcane.

\begin{abstract}
Photosynthetic responses of sugarcane leaves and gross photosynthetic efficiency of the crop
The gross photosynthetic efficiency of the sugarcane crop (Saccharum officinarum L.) is defined by the ratio of the daily value for gross photosynthesis of the $\operatorname{crop}(\mathrm{PB})$ to the active radiation absorbed daily by the crop. $\mathrm{PB}$ is estimated by the combination of leaf photosynthetic response, leaf irradiance and foliage structure. The photosynthetic responses to irradiation are obtained in an assimilation chamber (fig. 1). Variation in maximum net assimilation rate $(\mathrm{Nm})$ for these curves with leaf age and crop age are given in figures 2 and 3 . Theoretical variations of gross photosynthetic efficiency with incident irradiation, foliage characteristics and leaf photosynthetic characteristics are given in figure 4.

The evolution of gross photosynthetic efficiency $\left(\varepsilon_{\mathrm{PB}}\right)$ for a crop during growth is drawn in figure 5. Dark respiration has been estimated to obtain net photosynthesis efficiency $\left(\varepsilon_{b}^{\prime}\right)$, which is compared with the efficiency of solar energy conversion into dry matter $\left(\varepsilon_{b}\right)$.
\end{abstract}

\section{INTRODUCTION}

La productivité d'un couvert végétal peut être exprimée par le rendement de sa conversion du rayonnement solaire en matière sèche (WASSINK, 1959 ; NICHIPOROVICH, 1967).

La teneur en éléments minéraux du matériel végétal est généralement assez faible pour qu'il soit possible de considérer l'accumulation journalière de matière sèche comme le bilan entre une fixation, due à l'activité photosynthétique pendant la phase diurne, et des " pertes », dues aux mécanișmes respiratoires sur l'ensemble du nycthémère.

Chez la canne à sucre (Saccharum officinarum L.), l'activité photosynthétique correspond à la photosynthèse brute (photorespiration nulle ou négligeable; plante en «C4»). La respiration de type « obscur » peut être supposée non inhibée à la lumière (CHAPMAN \& GRAHAM, 1974 ; RYLE 
et al.,1976) et rend compte de toutes les pertes d'énergie sur le cycle journalier ; ces pertes sont notamment fonction de l'intensité de la photosynthèse diurne (PENNING DE VRIES, 1972).

La conversion du rayonnement solaire en matière sèche par une culture de canne à sucre doit donc dépendre largement de sa photosynthèse brute $(\mathrm{PB})$.

De nombreux auteurs (DE WIT, 1965 ; DUNCAN et al., 1967 ; CHARTIER, 1969 ; GOUDRIAAN \& VAN LAAR, 1978) ont développé des modèles d'estimation de la photosynthèse d'un couvert végétal à partir des réponses photosynthétiques des feuilles à la lumière, de la structure de la végétation et des éclairements à différents niveaux dans la culture.

Nous avons appliqué la méthode de CHARTIER (1969) pour estimer la photosynthèse brute de cultures de canne à sucre en Guadeloupe. Les valeurs ainsi obtenues sont rapportées à l'énergie utile à la photosynthèse (gamme spectrale 400-700 nm) absorbée par la culture, pour définir une efficience de la photosynthèse brute $\varepsilon_{\mathrm{PB}}$.

Nous avons étudié les variations théoriques de cette efficience en donnant aux principaux paramètres nécessaires à son calcul les valeurs extrêmes rencontrées chez la canne à sucre. Nous avons également calculé l'évolution de $\varepsilon_{\mathrm{PB}}$ au cours du cycle d'une culture et essayé d'analyser ainsi les variations (mesurées) du rendement de la conversion du rayonnement solaire en matière sèche $\left(\varepsilon_{\mathrm{h}}=\right.$ rapport entre l'énergie potentielle chimique accumulée dans la matière sèche totale et le rayonnement utile à la photosynthèse absorbé par la culture).

Avant de présenter ces résultats, nous donnons aussi l'évolution de la réponse photosynthétique des feuilles à l'éclairement en fonction de l'âge de la feuille et de la culture ; ces données ayant été utilisées pour l'étude de $\varepsilon_{\mathrm{PB}}$.

\section{MATÉRIEL ET MÉTHODE}

Les mesures ont été effectuées sur différentes variétés de canne à sucre («PR 1059 », «HJ 5741 », «B6 4277 ») au laboratoire de Bioclimatologie (I.N.R.A.) du domaine Duclos, en Guadeloupe ( $16^{\circ}$ Nord, $62^{\circ}$ Ouest). Les cultures ont été conduites selon les normes classiques mais optimales (irrigation, fumure) définies pour la région (LEMAIRE \& ROBERT, 1973).

\section{A) Réponse photosynthétique d'une feuille}

La réponse photosynthétique d'une feuille est obtenue par la mesure des échanges de gaz carbonique entre l'air et un morceau de limbe situé dans une chambre d'assimilation analogue à celle de CHARTIER \& CHARTIER (1971). L'échantillon est placé dans une enceinte ventilée (environ $1 \mathrm{~m} \mathrm{~s}^{-1}$ ) et maintenue à une température moyenne de $28^{\circ} \mathrm{C}$, l'hygrométrie n'est pas contrôlée, mais reste toujours élevée (air extérieur, climat tropical humide). Un débit d'air connu (autour de $200 \mathrm{l} / \mathrm{h}^{-1}$ ) circule dans la chambre de façon à maintenir la différence de concentration du gaz carbonique entre l'entrée et la sortie (mesurée par analyseur infra-rouge Cosma, $0-500 \mathrm{vpm}$ ) inférieure à $50 \mathrm{vpm}$. Quatre lampes quartz-iode (Osram $1000 \mathrm{~W}$ ) permettent d'obtenir au niveau de la feuille (après traversée d'un filtre d'eau) 7 à 9 niveaux d'éclairement (selon les expériences) jusqu'à $2,4 \cdot 10^{-}{ }_{3}$ $\mathrm{Em}^{-2} \mathrm{~s}^{-1}$ (mesurés par cellule Lambda « quantum sensor »).

La feuille étudiée est prise au champ, tôt le matin ; on prélève (sous l'eau) dans sa partie médiane, un morceau (environ $20 \mathrm{~cm}$ de long) qui est placé horizontalement dans la chambre, la face supérieure dirigée vers la lumière et alimentée en eau par les sections. La surface de cet échantillon est mesurée par un planimètre électronique (Metraplan) et, dans certains cas, nous avons déterminé aussi ses facteurs de réflexion et de transmission pour la gamme des radiations visibles $(400-700 \mathrm{~nm})$ à l'aide d'un dispositif à miroir hémisphérique analogue à celui de SINCLAIR \& THOMAS (1970).

Ce morceau de feuille est d'abord soumis à un "éclairement " moyen $\left(0,5 \cdot 10^{-3} \mathrm{Em}^{-2} \mathrm{~s}^{-1}\right)$ jusqu'à l'équilibre (environ 30 à $45 \mathrm{mn}$ ) puis aux "éclairements » plus faibles $\left(0,35 \cdot 10^{-3}\right.$ et $\left.0,15 \cdot 10^{-3} \mathrm{Em}^{-2} \mathrm{~s}^{-1}\right)$ pour revenir aux « éclairements " plus élevés $\left(0,65 \cdot 10^{-3}, 1,25 \cdot 10^{-3}, 1,85 \cdot 10^{-3}\right.$, $2,4 \cdot 10^{-3} \mathrm{Em}^{-2} \mathrm{~s}^{-1}$ ) et finir par l'obscurité (mesure de $\mathrm{R}_{\mathrm{o}}$ ).

La réponse de la feuille dépend de la zone de prélèvement du limbe et de la face éclairée. Nous avons noté des différences d'intensité de la photosynthèse de la partie médiane de $+15 \mathrm{p}$. cent par rapport à la base et de 15 p. 100 par rapport à l'extrémité du limbe. Nous avons supposé que cette partie médiane donnait une "réponse moyenne » de la feuille entière.

L'assimilation nette de la face supérieure semble (quelques mesures seulement) toujours plus élevée que celle de la face inférieure, mais l'écart est assez faible (environ 5 p. 100)

Les courbes donnant l'assimilation nette de l'unité de surface $\mathrm{N}$ (en $\mathrm{kg} \mathrm{CO}_{2} \mathrm{~m}^{-2} \mathrm{~s}^{-1}$ ) ainsi mesurée, en fonction du rayonnement utile à la photosynthèse incident PAR (en $\mathrm{E} \mathrm{m}^{-2} \mathrm{~s}^{-1}$ ) présentent toutes une même forme (fig. 1) qui

Assimilation nette $\mathrm{N}$

$\left(10^{-6} \mathrm{KgCO}_{2} \mathrm{~m}^{-2} \mathrm{~s}^{-1}\right)$

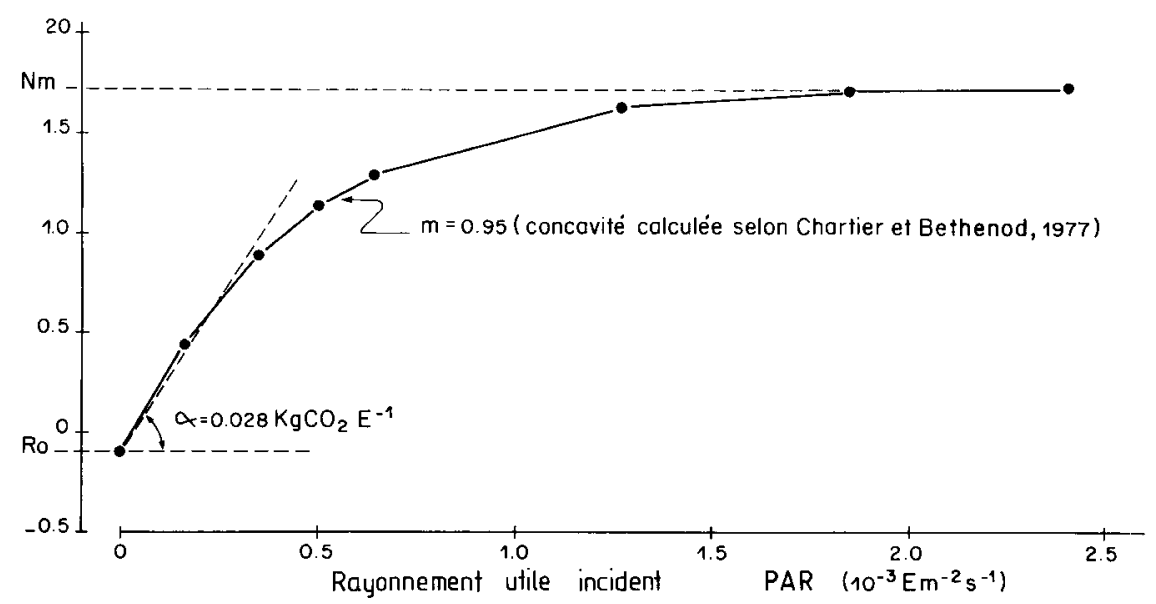

Figure 1

Assimilation nette de l'unité de surface de feuille $\mathrm{N}$ (en $\mathrm{kg} \mathrm{CO}_{2} \mathrm{~m}^{-2} \mathrm{~s}^{-1}$ ) en fonction du rayonnement utile à la photosynthèse incident PAR (en $E m^{-2} s^{-1}$ ).

Net assimilation rate $N$ versus photosynthet cally active radiation $P A R$. 
correspond à celle déjà observée sur de nombreuses espèces.

Ces courbes peuvent être caractérisées par :

- $R_{o}$, la valeur de la respiration à l'obscurité,

- $\alpha$, la pente de la partie linéaire de la courbe, qui représente le rendement lumineux maximal de la photosynthèse,

- $\mathrm{m}$, la concavité de la courbe selon l'ajustement de CHARTIER \& BETHENOD (1977),

- $\mathrm{N}_{\mathrm{m}}$, la valeur maximale de l'assimilation nette pour l'éclairement de saturation.

Parmi ces paramètres, $\mathrm{N}_{\mathrm{m}}$ est celui qui est déterminé avec le plus de précision et qui présente les variations les plus importantes en fonction de l'âge de la feuille et de la culture. De plus, sa valeur correspond aussi à la saturation de la photosynthèse par rapport à la concentration en gaz carbonique (pour une vingtaine de mesures effectuées sur plusieurs variétés nous avons obtenu une concentration de saturation sous fort éclairement de $290 \pm 12 \mathrm{vpm}$ ).

Nous avons donc retenu $\mathrm{N}_{\mathrm{m}}$ pour montrer l'évolution de la réponse photosynthétique des feuilles en fonction de leur âge et de celui de la culture.

Les autres paramètres $R_{0}$ et $m$ ne présentent pas de variations systématiques alors que $\alpha$ évolue comme $\mathrm{N}_{\mathrm{m}}$; cependant ses valeurs sont peu précises et leur détermination critiquable : ajustement linéaire sur des valeurs de $\mathrm{N}$ correspondant à des éclairements trop élevés (non-linéarité de la réponse).

La feuille est généralement positionnée par rapport au fouet foliaire (sommet de la tige) : la feuille numérotée 1 (ou dite de rang 1) correspond alors à la $1^{\text {re }}$ feuille entièrement déroulée (okréa visible, VAN DillewiJn, 1954). Cependant pour le même rang, l'âge de la feuille peut varier ; nous avons donc essayé d'estimer cet âge avec plus de précision.

A l'intérieur du fouet foliaire, la feuille la plus jeune suffisamment grande pour être manipulable $(10$ à $15 \mathrm{~cm}$ de longueur environ) est repérée par un fil de couleur (celle-ci codifie la date). Cette opération est effectuée à différents stades, sur une vingtaine de pieds présentant à peu près le même état de développement (hauteur, nombre de feuilles visibles). Pour noter précisément le moment où le limbe se déroule complètement, il aurait fallu suivre la progression des feuilles marquées à l'intérieur du fouet foliaire. Le nombre des observations nécessaires étant trop important, il ne nous a pas été possible de les effectuer de façon systématique. Toutefois, un travail préliminaire effectué sur des repousses de diverses variétés et pour une vingtaine de feuilles a montré que le temps moyen pour atteindre le déroulement à partir du marquage était de $21 \mathrm{j}$ (avec des extrêmes de 20 à $24 \mathrm{j}$ ). Nous avons admis cette durée pour toutes nos mesures ; ainsi, pour chaque feuille prélevée, la date exacte du marquage était donnée par le repère de couleur et la date de son déroulement complet était estimée en ajoutant 21 jours.

\section{B) Efficience de la photosynthèse brute de la culture}

La méthode de calcul de la photosynthèse brute (PB) de la culture est analogue à celle de CHARTIER (1969). Les éclairements à différents niveaux sont obtenus selon BON HOMME \& VARLET-GRANCHER (1977). Les données relatives à la structure du couvert végétal : indice foliaire $(\mathrm{F})$ et sa répartition verticale (profil), inclinaison moyenne des feuilles... sont tirées de VARLET-GRANCHER et al. (1980). La variation de la photosynthèse brute par unité de surface foliaire $\mathrm{P}$ en fonction de l'éclairement $\mathrm{E}$ est décrite par la même loi que CHARTIER \& BETHENOD (1977), en donnant aux paramètres caractéristiques $\left(\alpha, \mathrm{m}, \mathrm{P}_{\mathrm{m}}\right)$ les valeurs obtenues sur les courbes expérimentales $N=f(E)$ (après transformation $P_{m}=N_{m}+R_{o}$ ). Nous avons utilisé une même réponse par couche de végétation de $50 \mathrm{~cm}$ d'épaisseur (celle de la feuille jugée arbitrairement la plus représentative d'après des silhouettes de plantes), l'inclinaison des feuilles est supposée constante pour toute la couche (correspond à l'inclinaison moyenne mesurée) et l'azimut aléatoire.

L'efficience $\varepsilon_{\mathrm{PB}}$ est obtenue à partir des valeurs de PB et de l'énergie utile à la photosynthèse absorbée par la végétation pendant le même temps et sur la même surface de sol, calculée selon VARLET-GRANCHER \& BONHOMME (1979).

En fait, la méthode de calcul présentée ci-dessus s'applique à des valeurs instantanées ; or, pour notre démarche, il nous importe de connaître les variations journalières de l'efficience photosynthétique brute. Au cours de la journée, même en supposant que la réponse photosynthétique des feuilles reste constante, $\varepsilon_{\mathrm{PB}}$ varie avec la hauteur du soleil, les rayonnements solaires directs et diffus. Si l'on veut connaître la valeur journalière de l'efficience photosynthétique en fonction de l'énergie journalière incidente, l'intégration est difficile car à un rayonnement journalier plus faible correspond une part de rayonnement diffus plus importante, etc..

Nous avons utilisé les lois établies par ORGILL \& HoLLANDS (1977) et PERRIN DE BRICHAMBAUT (1976) pour simuler la variation au cours de la journée des rayonnements diffus (D) et direct (S) à partir de la seule valeur journalière du rayonnement global $(\mathrm{G})$. Le tableau 1 montre que les efficiences obtenues avec des valeurs moyennes des caractéristiques de la culture et de la feuille, calculées pour quelques journées où $S$ et $D$ étaient mesurés en valeurs horaires ou estimés à partir de $\mathrm{G}$ journalier, sont du même ordre de grandeur.

TABLEAU 1

Valeurs journalières de l'efficience de la photosynthèse brute $\varepsilon_{P B}$ (p. 100) calculées à partir des évolutions des rayonnements directs (S) et diffus (D), estimées ou mesurées, et pour différents types de journée

Daily value of gross photosynthesis efficiency $\varepsilon_{P B}(p .100)$ for estimated or measured variations of diffuse D and direct $S$ solar irradiation during the day, and for different sky conditions

\begin{tabular}{|c|c|c|c|c|c|c|c|c|}
\hline Type de journée $\mathrm{D} / \mathrm{G}=$ & 0,26 & 0,35 & 0,40 & 0,51 & 0,56 & 0,65 & 0.75 & 1,0 \\
\hline Valeurs de $\varepsilon_{\mathrm{PB}}$ pour $\mathrm{D}$ et $\mathrm{S}$ mesurés & 7,7 & 7,7 & 9,1 & 9,6 & 9,4 & 10,5 & 11,3 & 12,3 \\
\hline Valeurs de $\varepsilon_{\mathrm{PB}}$ pour $\mathrm{D}$ et $\mathrm{S}$ estimés & 7,1 & 7,5 & 8,5 & 9,4 & 10,3 & 10,7 & 11,6 & 12,2 \\
\hline
\end{tabular}




\section{RESULTATS ET DISCUSSIONS}

\section{A) Variations de la réponse photosynthétique des feuilles}

La figure 2 montre l'évolution des valeurs de $N_{m}$ en fonction de l'âge de la feuille (le déroulement du limbe hors du fouet foliaire est indiqué sur l'axe des abscisses par la lettre D) et de l'âge de la culture (en jours après plantation), pour la variété «PR $1059 »$.
Bien que les mesures ne recouvrent pas toujours totalement toute la durée de vie des feuilles étudiées, il est possible de distinguer 2 séries de réponses.

Pour les courbes numérotées de 1 à 6 , les valeurs de $\mathrm{N}_{\mathrm{m}}$ passent par un maximum 15 à $20 \mathrm{j}$ après le déroulement du limbe puis diminuent assez rapidement vers un niveau relativement constant marquant ainsi un «épaulement » dans l'évolution, enfin $\mathrm{N}_{\mathrm{m}}$ chute très vite avec le dessèchement du limbe.

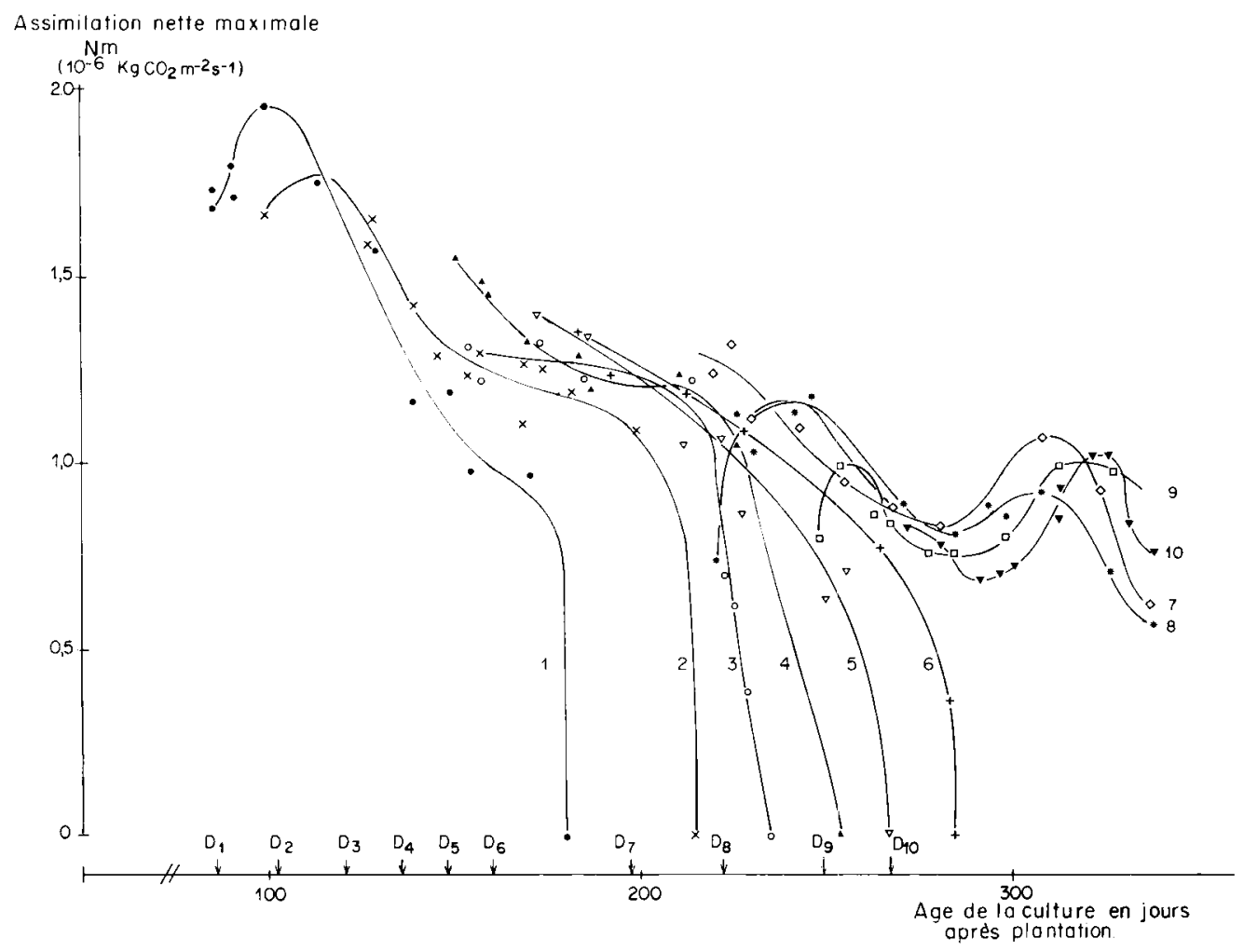

Figure 2

Evolution de l'assimilation nette maximale de l'unité de surface de feuille' $N_{m}$ pour différentes feuilles au cours du développement de la culture (lissage manuel).

Evolution of maximum net assimilation rate $N_{m}$ for different leaves during crop cvcle. The curves have been handle fitted.

Pour les feuilles 7 à 10 , les courbes diffèrent par la présence de 2 pics. Le $1^{\text {er }}$ correspond au maxima des courbes précédentes alors que le $2^{\mathrm{e}}$ apparaît à un âge variable de la feuille (entre 40 et $110 \mathrm{j}$ après déroulement) mais à peu près au même stade de développement de la culture ( 310 à $330 \mathrm{j}$ après plantation).

Les valeurs de ces maxima et des premiers pics diminuent fortement (de $2.10^{-6}$ à $1.10^{-6} \mathrm{~kg} \mathrm{CO}_{2} \mathrm{~m}^{-2} \mathrm{~s}^{-1}$ ) avec le vieillissement de la culture, atténuant ainsi l'importance des variations de $\mathrm{N}_{\mathrm{m}}$ durant la vie de chaque feuille.

Ces évolutions de $N_{m}$ ne peuvent être analysées à partir de nos mesures, cependant on peut rapprocher la forme des courbes 1 à 6 de celles obtenues par LUDLOW \& WILSON (1971b) sur des espèces voisines (Sorghum almunosorgho et Pennisetum purpureum).

Ces auteurs situent la valeur maximale de $\mathrm{N}_{\mathrm{m}}$ avec la fin de l'expansion foliaire (qui pour ces especes est atteint peu de temps après le déroulement du limbe) et montrent que la diminution de $\mathrm{N}_{\mathrm{m}}$ est surtout liée à l'augmentation de la résistance à la diffusion du gaz carbonique au niveau des stomates $\left(r_{s}\right)$ et, dans une moindre mesure, à celle des stomates aux sites de carboxylation $\left(\boldsymbol{r}_{\mathrm{m}}\right)$.

Sur canne à sucre, la réponse photosynthétique de la feuille a été étudiée par rapport à sa position sur la tige ; Bull (1969) montre le rôle prépondérant de $r$ dans la diminution de $\mathrm{N}_{\mathrm{m}}$ avec l'augmentation de son rang.
L'effet du vieillissement des plantes est aussi mis en évidence par l'évolution du $\mathrm{N}_{\mathrm{m}}$ pour des feuilles occupant le sommet de la tige (rang 1 à 3 ) au cours du cycle de la culture. La figure 3 donne les résultats ainsi obtenus pour 3 variétés ; la courbe correspondant à la variété «B 64277 » est analogue à celles décrites par HARTT \& BURR (1967) et par BULL (1971) alors que l'évolution de la variété «HJ 5741 » est analogue à celle de KORTSCHAK \& FORBES (1969).

Ces variations de $\mathrm{N}_{\mathrm{n}}$ ont été attribuées à une évolution de la résistance stomatique (BULL, 1971) qui pourrait être liée selon cet auteur à celle du rayonnement incident. Mais les variations indiquées par BULL sont beaucoup plus importantes que celles que nous avons en Guadeloupe où le rayonnement solaire a un niveau toujours assez élevé (entre 30 et $60 \mathrm{E} \mathrm{m}^{2} \mathrm{j}^{1}$ ). L'hypothèse d'une régulation de la photosynthèse liée à l'accumulation du saccharose dans la tige et, par conséquent, d'un « engorgement » au niveau de la feuille, émise par HARTT (1963), a été infirmée par les travaux de WALDRON et al. (1967).

Les paramètres expliquant l'effet du vieillissement de la plante sur la réponse des jeunes feuilles ne semblent pas encore bien établis.

Nous retiendrons que cet effet peut varier avec le génotype et que la comparaison des réponses photosynthéti- 


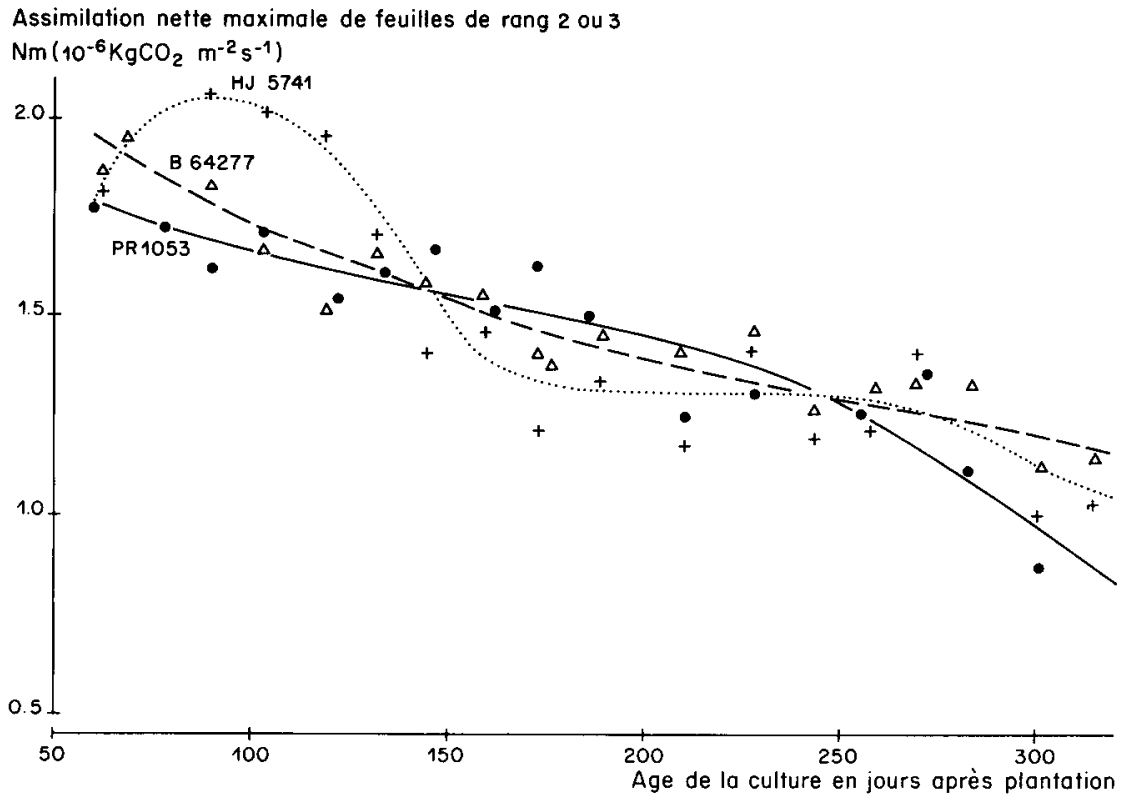

Figure 3

Evolution de l'assimilation nette maximale $N_{m}$ de feuilles de rang 2 ou 3 en fonction de l'âge de la culture en jours après plantation. + variété "HJ 5741 "

- variété «PR 1059 »

$\triangle$ variété « $B 64277$ »

Evolution of maximum net assimilation rate $N_{m}$ for leaves of rank 2 or 3 versus crop age in days after planting.

ques des feuilles de différentes variétés doit tenir compte du stade de développement des cultures.

La présence du $2^{\mathrm{e}}$ pic dans les évolutions des feuilles 7 à 10 de la figure 2 est également assez difficile à expliquer. Toutefois on peut rapprocher peut-être ce type de courbe avec les évolutions de la réponse photosynthétique de feuilles soumises à des variations de leur éclairement en cours de culture, observées par divers auteurs (HODGKINSON, 1974 ; WOLEDGE, 1971 ; BONHOMME et al., 1977) sur d'autres espèces.

Au cours du développement de la culture, la surface des nouvelles feuilles diminue (VAN DILLEWIJN, 1954) ; l'indice foliaire au-dessus d'une feuille de rang 9 par exemple passe de 3,2 à 2,2 entre 270 à $340 \mathrm{j}$ pour la variété «PR 1059 ». Les feuilles jeunes, à cette période, ont une disposition plus verticale (VARLET-GRANCHER et al., 1980) et forment une couche supérieure qui laisse mieux passer la lumière. Enfin cette période correspond aussi à une augmentation du rayonnement incident.

Ces différentes modifications pourraient provoquer une augmentation notable de l'éclairement des feuilles de rang 5 à 10 qui expliquerait alors l'apparition du $2^{\mathrm{e}}$ pic des courbes 7 à 10

\section{B) Variations théoriques de l'efficience de la photosynthèse brute de la culture $\varepsilon_{\mathrm{PB}}$}

Nous avons étudié l'influence de l'intensité du rayonnement incident, des paramètres caractéristiques de la structure de la végétation et des potentialités photosynthétiques des feuilles sur l'efficience $\varepsilon P B$, en donnant à ces facteurs une gamme de valeurs correspondant à celle observée dans les cultures étudiées.

La variation des valeurs journalières de $\varepsilon_{\mathrm{PB}}$ avec l'intensité du rayonnement utile à la photosynthèse (PAR) reçu par le couvert végétal est donnée sur la figure $4 \mathrm{a}$ (courbe 1) pour une culture bien développée (indice foliaire de 4); la diminution de $\varepsilon_{\mathrm{PB}}$ devient importante lorsque le PAR journalier est supérieur à $30 \mathrm{Em}^{-2}$ environ. Ce résultat correspond à une augmentation linéaire de la photosynthèse brute PB jusqu'à ce niveau d'éclairement (soit en valeur instantanée environ $1,2 \cdot 10^{-3} \mathrm{E} \mathrm{m}^{-2} \mathrm{~s}^{-1}$ ) puis à un plateau de saturation. Les mesures faites sur plantes en pots (WALDRON et al., 1967 ; GLOVER, 1974) ou sur culture « in situ » (CHU Chang CHI, 1970) sont en bon accord avec ce résultat. Cependant, pour d'autres espèces du même type biochimique (plante en « $\mathrm{C} 4$ »), l'éclairement de saturation de la photosynthèse brute semble être encore plus élevé (DE WIT et al. 1978, sur maïs).

Le rayonnement étant l'un des facteurs les plus variables, nous présenterons l'influence des autres paramètres par leur action sur la variation de $\varepsilon_{\mathrm{PB}}$ avec le PAR incident.

La structure de la végétation est caractérisée par l'indice foliaire $F$ et l'inclinaison moyenne (i) des feuilles. La figure 4a donne l'évolution de $\varepsilon_{\mathrm{PB}}$ pour des inclinaisons de 45 et $65^{\circ}$ avec des indices foliaires de 0,5 et 4 . Les écarts les plus importants sont obtenus pour des éclairements moyens; les courbes $4\left(i=45^{\circ}, F=0,5\right)$ et $1\left(i=65^{\circ}\right.$, $F=4,0$ ) indiquent l'effet théorique maximum des variations de structure du couvert végétal au cours du cycle (les cultures plus âgées ont des feuilles plus verticales, VARLETGRANCHER et al., 1980). Cet effet apparaît donc assez faible pour la canne à sucre, cependant l'influence de la géométrie semble beaucoup plus marquée sur les valeurs instantanées de la photosynthèse brute et avec une gamme de variations de $i$ et F plus large (DUNCAN et al., 1967, KUROIWA, 1970). L'intégration journalière de la réponse des cultures atténue fortement l'influence de la géométrie de la végétation (Sinclair, 1971 ; GoUdriaAn \& VAN LAaR, 1978).

La figure 4 montre que l'influence des caractéristiques photosynthétiques des feuilles $\mathrm{P}_{\mathrm{m}}, \alpha$ et $\mathrm{m}$ sur $\varepsilon_{\mathrm{PB}}$ est très importante, mais dépend du niveau du rayonnement incident. Pour les faibles éclairements, l'effet de $\alpha$, (fig. 4b) est prépondérant (quasi-proportionnalité) alors que $\varepsilon_{\mathrm{PB}}$ dépend nettement du $\mathrm{P}_{\mathrm{m}}$ pour les rayonnements moyens et forts (fig. 4c). La concavité (m) des courbes de réponses $\mathrm{P}=\mathrm{f}(\mathrm{E})$, a un effet plus faible (pour la gamme de valeurs considérée) qui est surtout marqué aux éclairements moyens (fig. 4d). Ces résultats sont en accord avec ceux de nombreux auteurs (DE WIT, 1965; DE WIT et al., 1978; GOUDRIAN \& VAN LAAR, 1978) bien que peu de travaux considèrent l'effet de ces facteurs par rapport au niveau du rayonnement incident et pour des valeurs journalières de la photosynthèse brute.

Pour chaque feuille les caractéristiques photosynthétiques sont évidemment liées et leur valeur diminue avec la sénescence du limbe (cf. \$ III.A) ; l'efficience photosynthétique d'une culture âgée sera donc très faible. Cependant, pour la canne à sucre, le renouvellement des feuilles tout au 


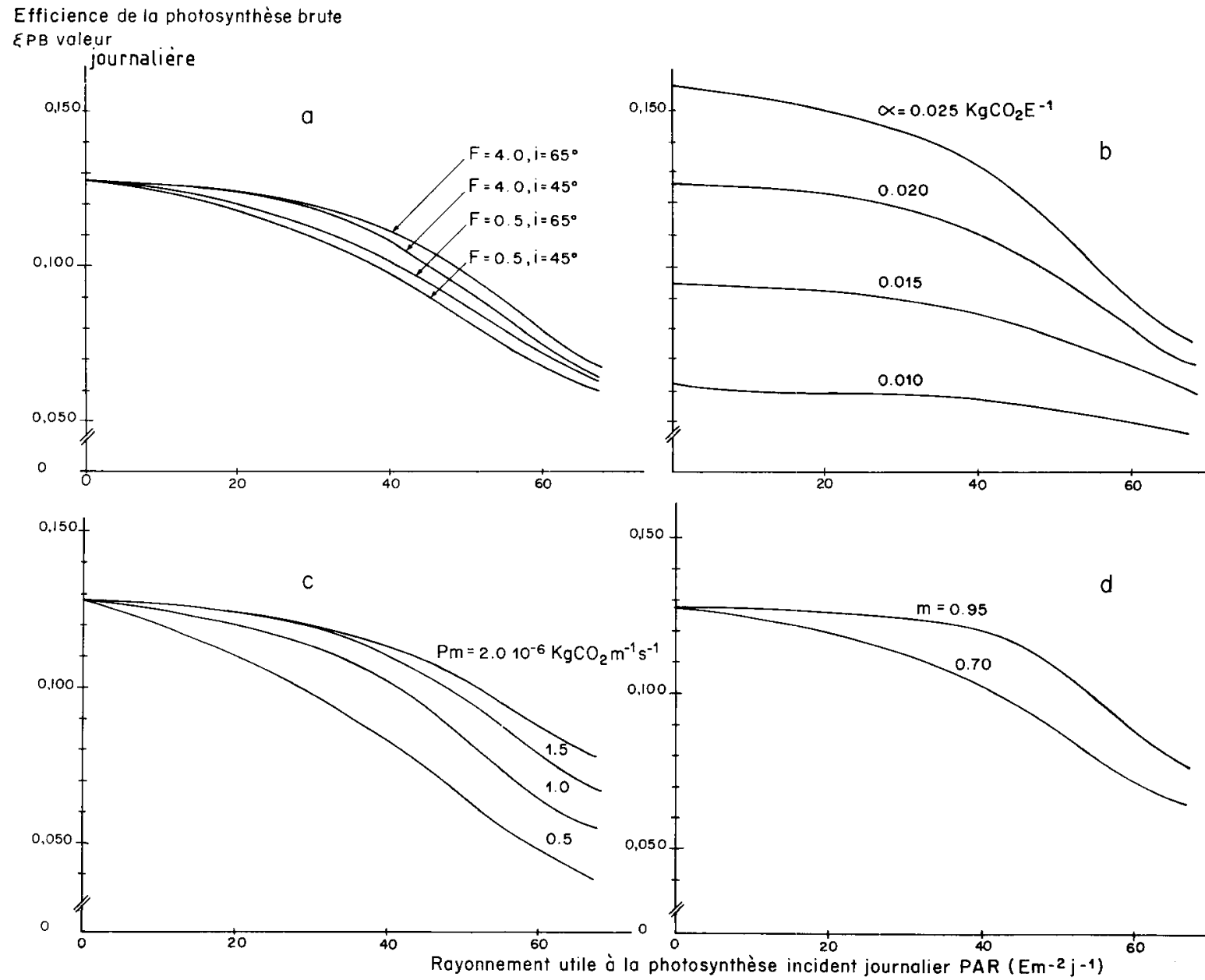

Figure 4

Efficience théorique de la photosynthèse brute $\varepsilon_{P B}$; valeurs journalières en fonction du rayonnement journalier utile à la photosynthèse reçu par la culture (PAR en Einstein $m^{-2} j^{1}$ ).

a) Influence de l'inclinaison des feuilles et de lindice foliaire de la culture:

$P_{m}=1,510^{-6} \mathrm{~kg} \mathrm{CO}_{2} \mathrm{~m}^{-2} \mathrm{~s}^{-1}, \alpha: 0,020 \mathrm{~kg} \mathrm{CO} \mathrm{E}^{-1}, \mathrm{~m}=0,90$.

b) Influence des caractéristiques photosynthétiques des feuilles : $\alpha P_{m}=1,510^{-6} \mathrm{~kg}_{\mathrm{CO}} \mathrm{m}^{2} \mathrm{~s}^{-1}, \mathrm{~m}=0,90, \mathrm{~F}=4,0, i=65^{\circ}$.

c) Influence des caractéristiques photosynthétiques des feuilles: $P_{m} \alpha=0,020 \mathrm{~kg} \mathrm{CO} E^{-1}, m=0,90, F=4,0, i=65^{\circ}$.

d) Influence des caractéristiques photosynthétiques des feuilles: $m P_{m}=1,510^{6} \mathrm{~kg}^{2} \mathrm{CO}_{2} \mathrm{~m}^{-2} \mathrm{~s}^{-1}, \alpha=0,020 \mathrm{~kg} \mathrm{CO}_{2} E^{-1}, F=4,0, i=65^{\circ}$.

Variations of theoretical daily value of gross photosynthesis efficiency $\varepsilon_{P B}$ versus incident photosynthetically active radiation; influence of : a) leaf inclination $i$ and leaf area index $F$,

b) leaf maximum photosynthesis efficiency $\alpha$,

c) leaf maximum gross photosynthesis rate $P_{m}\left(P_{m}=N_{m}+R\right)$.

d) leaf photosynthesis characteristic $m$ (« curvature" of photosynthetic response to irradiance, CHARTIER \& BETHENOD, 1977).

long du cycle compense partiellement cet effet ; ces caractéristiques dépendant aussi de l'âge du couvert végétal.

Le rendement de la photosynthèse brute de la culture, pour la canne à sucre, dépend donc surtout du niveau du rayonnement incident et des réponses photosynthétiques des feuilles.

\section{C) Évolution de l'efficience de la photosynthèse brute au cours du développement de la culture}

L'efficience $\varepsilon_{\mathrm{PB}}$ a été calculée pour différents stades au cours du développement de la culture en donnant à chaque paramètre $\left(\mathrm{i}, \mathrm{F}, \mathrm{N}_{\mathrm{m}} \ldots\right)$ les valeurs mesurées sur la variété «PR 1059 » (fig. 5).

Pendant les premières phases de la croissance (jusqu'à $150 \mathrm{j}$ environ), les variations de tous les paramètres considérés conduisent à une augmentation de l'efficience $\varepsilon_{\mathrm{PB}}$ (diminution du PAR incident, augmentation de i.et $\mathrm{F}$ ) alors que la chute de $\varepsilon_{\mathrm{PB}}$ à partir de $200 \mathrm{j}$ correspond surtout à l'effet de l'âge de la culture sur la réponse photosynthétique des feuilles.

L'efficience de la photosynthèse brute pour la durée de la culture résulte évidemment de l'évolution de $\varepsilon_{\mathrm{PB}}$ par rapport à celle du rayonnement utile à la photosynthèse absorbé par la végétation $\left(\mathrm{PAR}_{\mathrm{a}}\right)$; ainsi l'influence de la chute de $\varepsilon_{\mathrm{PB}}$ en fin de cycle est d'autant plus marquée que le rayonnement absorbé $\left(\mathrm{PAR}_{\mathrm{a}}\right)$ est plus élevé (interception maximale et augmentation du rayonnement incident). La valeur de $\varepsilon_{\mathrm{PB}}$ pour la durée du cycle de culture $\left(\varepsilon_{\mathrm{PB}}=0,103\right)$ donne le rendement photosynthétique maximum de la variété considérée pour les conditions climatiques (rayonnement) de la saison.

Le niveau de ce rendement est indicatif puisque le calcul de la photosynthèse brute est très simplifiée et ne prend pas en compte tous les facteurs.

La méthode de calcul utilisée ici ne tient pas compte de l'influence de la température sur les réponses photosynthétiques (LUDLOW \& WILSON, 1971a ; MORE HERRERO et al., 


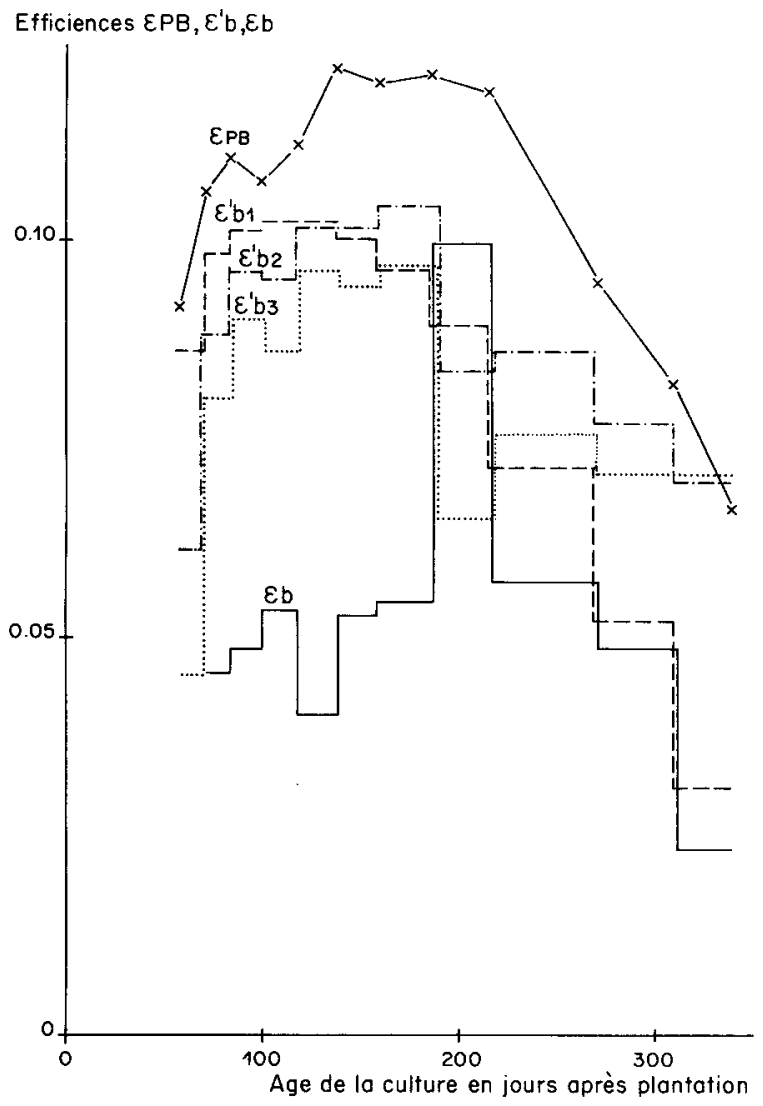

Figure 5

Evolution des efficiences, $\varepsilon_{P B}, \varepsilon_{R D}, \varepsilon_{b}^{\prime}=\varepsilon_{P B}-\varepsilon_{R D}$ et $\varepsilon_{b}$ au cours du cycle de la culture en jours après la plantation.

$\varepsilon_{b 1}^{\prime}: R D$ calculée selon GLOVER (1973).

$\varepsilon_{b 2}: R D$ calculée selon MC CREE (1974).

$\varepsilon_{b 3}: R D$ calculée selon RUGET (comm. pers. 1980).

Evolution of crop gross photosynthesis efficiency $\varepsilon_{P B}$, crop dark respiration efficiency $\varepsilon_{R D}$ and crop net photosynthesis efficiency (estimated $\varepsilon_{b}^{\prime}=\varepsilon_{P B}-\varepsilon_{R D}$ or measured $\varepsilon_{b}$ ).

$\varepsilon_{b 1}^{\prime}$ : crop dark respiration calculated by GLOVER (1973).

$\varepsilon_{b 2}:$ crop dark respiration calculated by MC CREE (1974).

$\varepsilon_{b 3}$ : crop dark respiration calculated by RUGET (pers. comm., 1980).

1980). Pour la canne à sucre, WALDRON et al. (1967) obtient un Q10 de 1,1 pour l'efficience de la photosynthèse nette (par rapport au PAR incident et sur la phase diurne) de plantes en pot placées à l'extérieur et dans la gamme 5 à $34{ }^{\circ} \mathrm{C}$.

Étant données les variations de la température au cours de l'année (moyenne journalière décadaire entre 21 et $26^{\circ} \mathrm{C}$ ), l'action de ce facteur semble pouvoir être négligée. De plus, l'effet de la température de l'air paraît parfois plus faible pour les plantes cultivées en conditions naturelles que celles obtenues en chambre de croissance généralement utilisées pour établir les lois d'action de ce facteur (DE WIT et al., 1978).

Les cultures sont supposées bien alimentées en eau (irrigation à la plantation puis pluviométrie satisfaisante) et en éléments minéraux (fumure optimale par rapport au rendement agronomique). Bien que l'activité photosynthétique puisse varier au cours de la journée, même lorsque l'alimentation en eau de la plante est satisfaisante (CHU CHANG CHI, 1970 ; CoWAN \& FARQuHaR, 1976 ; Robelin \& MAUGET, 1977), de telles variations n'ont pas été introduites dans nos calculs.

Malgré ces réserves sur la validité du calcul de $\varepsilon_{\mathrm{pB}}$, nous avons comparé son évolution (fig. 5) à celle du rendement de la conversion du rayonnement utile à la photosynthèse absorbé par la végétation en matière sèche : $\varepsilon_{\mathrm{b}}$, obtenue à partir des courbes de croissance en matière sèche totale et de la mesure du rayonnement absorbé (VARLET-GRANCHER et al., 1981).

Les variations de $\varepsilon_{\mathrm{b}}$ ne semblent pas dépendre étroitement de $\varepsilon_{\mathrm{PB}}$; comme $\varepsilon_{\mathrm{b}}$ résulte du bilan entre la photosynthèse brute et la respiration, c'est à cette dernière composante que l'on doit alors attribuer un rôle prépondérant dans l'évolution de $\varepsilon b$.

La différence entre $\varepsilon_{\mathrm{PB}}$ et $\varepsilon_{\mathrm{b}}$ représente l'intensité théorique de la respiration $R_{D}$ rapportée à l'énergie absorbée $\left(\mathrm{PAR}_{\mathrm{a}}\right)$, mais pour analyser les variations de $\varepsilon_{\mathrm{b}}$ il paraît nécessaire de mesurer ou d'estimer RD de manière indépendante.

Nous avons utilisé les ajustements établis par GLOVER (1973) sur canne à sucre, donnant le flux de gaz carbonique respiré (RD), à partir de la biomasse (W) et de la température de l'air $\mathrm{T}(\mathrm{RD}=2.069 \log \mathrm{T}-2,1607 \mathrm{~W})$. Après transformation en son équivalent de matière sèche puis en énergie (en donnant à cette matière sèche le même équivalent calorifique que celui de la plante entière pour la période considérée), RD est rapportée à l'énergie absorbée (PAR $\left.{ }_{a}\right)$. On obtient ainsi l'équivalent d'une efficience $\varepsilon_{\mathrm{RD}}$ pour la respiration. Cependant, la fonction de GLOVER ne tenant pas compte de la respiration de croissance, nous avons aussi utilisé les résultats de Mc CREE (1974), obtenus sur sorgho en conditions chaudes $\left(\mathrm{T}=30^{\circ} \mathrm{C}\right)$ :

$$
\mathrm{RD}=0,41 \Delta \mathrm{W}+0,013 \mathrm{~W},
$$

et de RUGET (comm. pers., 1980), sur maïs en culture artificielle sous climat constant et chaud $\left(22^{\circ} \mathrm{C}\right)$ :

$$
\mathrm{RD}=0,64 \Delta \mathrm{W}+0,0059 \mathrm{~W} \text {, }
$$

$\Delta \mathrm{W}$ représentant l'accroissement de matière sèche et $\mathrm{W}$ sa valeur moyenne sur l'intervalle considéré.

Les valeurs ainsi obtenues pour $\varepsilon_{\mathrm{RD}}$ et celles de $\varepsilon_{\mathrm{PB}}$ permettent d'estimer le rendement de la conversion du rayonnement absorbé en matière sèche $\varepsilon_{b}^{\prime}=\varepsilon_{\mathrm{PB}}-\varepsilon_{\mathrm{RD}}$ qui peut être comparé au rendement mesuré $\varepsilon b$ (fig. 5).

Pour l'ensemble de la durée du cycle de culture, les valeurs estimées respectivement $(0,075 ; 0,086$ et 0,078$)$ surestiment toujours très largement (au minimum de 30 p. 100) la mesure $\left(\varepsilon_{\mathrm{b}}=0,057\right)$. Cependant les écarts entre $\varepsilon_{\mathrm{b}}^{\prime}$ et $\varepsilon_{\mathrm{b}}$ ne sont pas constants au cours du développement ; ils sont plus importants en début de culture (jusqu'à $180 \mathrm{j}$ environ) que pendant la période de maturité du couvert végétal.

Ces variations peuvent résulter seulement de l'accumulation des erreurs introduites dans les calculs (hypothèses simplificatrices, paramètres estimés) et faites sur les mesures $\left(\mathrm{PAR}_{\mathrm{a}}, \mathrm{N}_{\mathrm{m}}, \ldots\right)$ nécessaires pour obtenir $\varepsilon_{\mathrm{PB}}, \varepsilon_{\mathrm{RD}}, \varepsilon_{\mathrm{b}}^{\prime}$ et $\varepsilon_{\mathrm{b}}$.

Mais certaines procédures peuvent être plus particulièrement discutées : ainsi la comparaison de $\varepsilon_{\mathrm{b}}$ et $\varepsilon_{\mathrm{b}}^{\prime}$ oppose un bilan de matière sèche $\left(\varepsilon_{\mathrm{b}}\right)$ à un bilan de gaz carbonique $\left(\varepsilon_{\mathrm{PB}}-\varepsilon_{\mathrm{RD}}\right)$ qui est établi en supposant une teneur en carbone de la matière sèche, constante tout au long du cycle. Mais ces erreurs sont peut-être atténuées pour la canne à sucre car cette plante accumule essentiellement des carbohydrates. Nous avons aussi supposé que toutes les pertes d'énergie qui rendent compte du passage de $\varepsilon_{\mathrm{PB}}$ à $\varepsilon_{\mathrm{b}}$ sont représentées par la respiration alors que certains processus comme la réduction des nitrates pourraient être directement liés à la photosynthèse (BEEVERS \& HAGEMAN, 1969) et ne pas être pris en compte dans les mesures du flux de gaz carbonique respiré. De plus, pour calculer $\varepsilon_{R D}$, nous supposons que la quantité d'énergie «perdue»par la 
respiration est proportionnelle au flux de gaz carbonique dégagé alors que la nature des composés concernés dans les mécanismes respiratoires peut conduire à des variations importantes (PENNING DE VRIES, 1972).

Enfin, si l'on admet que les estimations de $\varepsilon_{\mathrm{PB}}$ et $\varepsilon_{\mathrm{b}}$ sont assez réalistes, la comparaison des évolutions de $\varepsilon_{\mathrm{b}}$ et $\varepsilon_{\mathrm{b}}^{\prime}$ pourrait aussi suggérer une régulation interne de la photosynthèse au cours du cycle. Ainsi $\varepsilon_{\mathrm{b}}^{\prime}$ représenterait une efficience potentielle de la culture considérée, qui ne serait atteinte $\left(\varepsilon_{\mathrm{b}}^{\prime}=\varepsilon_{\mathrm{b}}\right)$ que pour certaines périodes au cours de la croissance et du développement du couvert végétal, en particulier lorsque la " demande » en assimilats (ici période du « remplissage » des tiges) est très forte (effet «puitssource » de WARREN-WILSON, 1972).

\section{CONCLUSION}

La variabilité de la réponse photosynthétique de la feuille de la canne à sucre avec son vieillissement et celui de la culture avait déjà été signalée par différents auteurs (HARTT \& BURR, 1967 ; BULL, 1971) mais n'avait jamais été étudiée en séparant nettement ces 2 effets.

La forme des évolutions de la réponse photosynthétique des courbes 1 à 6 de la figure 2 est peut-être généralisable à l'espèce car elle présente des concordances avec les résultats obtenus par d'autres auteurs. Mais les variations (courbes 7 à 11) en relation éventuelle avec des modifications de la structure du feuillage et du rayonnement incident nécessiteraient une confirmation expérimentale sur d'autres cultures pour différentes variétés et conditions climatiques.

L'efficience de la photosynthèse brute varie de façon assez importante au cours du cycle de la culture ; malgré leurs limites, nos calculs montrent que ces variations résultent surtout du rayonnement incident et des réponses photosynthétiques des feuilles.

Il serait intéressant d'étudier la croissance pour des cultures plantées à différentes dates permettant de faire concorder la période de plus faible rayonnement avec les réponses photosynthétiques des feuilles les plus élevées (début de croissance). La pratique dite de "grande plantation » en Guadeloupe correspond à un décalage de la date de plantation (pour des raisons d'ajustement entre les récoltes et la durée d'activité des usines de traitement) dont l'optimisation serait peut être ainsi rendue possible.

Un grand nombre d'auteurs ont essayé de modéliser l'accumulation de matière sèche d'un couvert végétal à partir du bilan de la fixation du gaz carbonique. Beaucoup d'entre eux se sont surtout attachés à estimer l'activité photosynthétique de la culture en combinant les réponses des feuilles à l'éclairement et la dispersion du rayonnement solaire dans la végétation.

Nous avons appliqué ici l'une de ces méthodes (CHAR TIER, 1969) à un cas relativemeñt simple : culture de canne à sucre (plante pérenne, mais en $1^{\text {ere }}$ année pas de phase reproductive, plante de type $« \mathrm{C}_{4}$ ", photorespiration nulle ou négligeable) soumise à un climat assez peu variable (rayonnement et température en particulier).

Cependant les résultats ainsi obtenus $\left(\varepsilon_{\mathrm{PB}}\right)$ expliquent mal les variations de la production de matière sèche $(\varepsilon b)$; les écarts entre $\varepsilon_{\mathrm{PB}}$ et $\varepsilon_{\mathrm{b}}$ sont très variables au cours du cycle de la culture et ne semblent pas pouvoir être totalement attribués aux pertes respiratoires $\left(\varepsilon_{\mathrm{RD}}\right)$. Toutefois l'estimation de $\varepsilon_{R D}$ est très difficile étant donné la rareté des travaux qui permettent de modéliser la respiration à l'échelle de la culture et sur l'ensemble de son cycle.

Il faut remarquer aussi que les nombreux travaux portant sur la simulation de la photosynthèse du couvert végétal ont essentiellement développé des modèles de pénétration du rayonnement solaire alors que l'intégration de la variabilité des réponses de la feuille n'a pas été étudiée. Il est cependant possible que la courbe de "réponse équivalente " de l'ensemble de la végétation soit beaucoup moins variable car elle résulte surtout de la réponse des feuilles d'âge ou de rang moyen dont les variations sont plus faibles (fig. 2) et qui représentent la plus grande partie de la surface foliaire.

De plus, certains types de régulation (en particulier les relations «puits-source") ne sont peut-être pas mis en évidence par cette méthode de caractérisation des réponses des feuilles. Ainsi la courbe obtenue pour $\varepsilon_{\mathrm{PB}}$ définirait le potentiel photosynthétique de la variété sous le climat considéré. Pendant la plus grande partie de son cycle la plante " travaillerait " donc en dessous de ce niveau potentiel ; la régulation de type «puits-source» n'agirait pas comme « activateur » de l'efficience photosynthétique mais permettrait à la plante de fonctionner avec son maximum d'efficacité. Ainsi l'efficience photosynthétique ne serait généralement pas un facteur limitant de la production de matière sèche.

Il nous semble que les modèles de photosynthèse de la culture basés sur la réponse des feuilles sont encore trop imprécis pour être utilisés dans l'étude de la productivité en matière sèche à l'échelle du champ. Les techniques de mesure de la fixation nette nycthémérale du gaz carbonique par le couvert végétal paraissent maintenant suffisamment fiables (PETERs et al., 1974 ; KANEMASU \& HIEBSCH, 1975 ; GOSSE comm. pers. 1980) pour permettre sa modélisation en fonction de l'énergie solaire absorbée (HoDGES et al., 1979) elle-même assez bien simulée par les nombreux modèles de pénétration des rayonnements (LEMEUR \& BLAD, 1974).

Cependant l'analyse de la réponse photosynthétique de la feuille reste nécessaire, non seulement comme l'un des niveaux privilégiés pour comprendre le passage entre le chloroplaste ou la cellule et l'ensemble du couvert végétal, mais également comme fonction physiologique pouvant permettre de caractériser des différences génotypiques ainsi que les effets de divers traitements (attaque parasitaire, toxicité de produits...)

Reçu le 20 décembre 1980. Accepté le 11 mars 1981.

\section{RÉFÉRENCES BIBLIOGRAPHIQUES}

Beevers L., Hageman R. H., 1969. Nitrate reduction in higher plants. Annu. Rev. Plant Physiol., 20, 495-522.

Bonhomme R., Varlet-Grancher C., 1977. Application aux couverts végétaux des lois de rayonnements en milieu diffusant. I. Etablissement des lois et vérifications expérimentales. Ann. agron. 28 (6), 567-582.

Bonhomme R., Varlet-Grancher C., Chartier M., Artis P., 1977. Utilisation de l'énergie solaire par une culture de Vigna sinensis.
IV. Influence de l'âge et des éclairements passés sur le potentiel photosynthétique des feuilles cotylédonaires. Ann. agron. 28 (2), 159-169.

Bull T. A., 1969. Photosynthetic efficiencies and photorespiration in Calvin cycle an C4-dicarboxylic acid plants. Crop Sci., 6, 726-729.

Bull T. A., 1971. The C4 pathway related to growth rates in sugarcane. In «Photosynthesis and photorespiration», M. D. 
Hatch, C. B. Osmond \& R. O. Slatyer Ed., Wiley Interscience, 68-74.

Chapman E. A., Graham D., 1974. The effect of light on the tricarboxylic acid cycle in green leaves. I. Relative rates of the cycle in the dark and the light. Plant Physiol., 53, 879-885.

Chartier P., 1969. Assimilation nette d'une culture couvrante. I. Détermination de l'assimilation nette de la culture à partir d'une analyse théorique. Ann. Physiol. veget. 11 (2), 123-159.

Chartier P., Bethenod O., 1977. La productivité primaire à l'échelle de la feuille. In "Les processus de la production végétale primaire» A. Moyse Ed., Gauthiers-Villars, Paris, 77-112.

Chartier M., Chartier P., 1971. Design of an air conditioned assimilation chamber for detached leaves. Photosynthetica. 5 (1), 74-75.

Chu Chang Chi, 1970. Solar radiation and photosynthesis of sugar-cane in the field. Taiwan Sugar, 27, 14-21.

Cowan J. R., Farquhar G. D., 1976. Stomatal function in relation to leaf metabolism and environment. 31th Symp. Soc. exp. Biol. 7-9 sept. 1976, 471-505.

Duncan W. G., Loomis R. S., Williams W. A., Hanau R., 1967. A model for simulating photosynthesis in plant communities. Hilgardia, 38, 4, 181-205.

Glover J., 1973. The dark respiration of sugar-cane and the loss of photosynthate during the growth of a crop. Ann. Bot. 37, 845-852.

Glover J., 1974. The rate of apparent photosynthesis of whole sugar-cane plants. Ann. Bot. 38, 909-920.

Goudriaan J., Van Laar H. H., 1978. Calculation of daily totals of the gross $\mathrm{CO}_{2}$ assimilation of leaf canopies. Neth. J. agric. Sci., 26, 373-382.

Hartt C. E., 1963. Translocation as a factor in photosynthesis. Naturwissenschaften, 21, 666-667.

Hartt C. E., Burr G. O., 1967. Factors affecting photosynthesis in surgar-cane. Proc. int. Soc. Sugar-cane Technol., 12, 590-609.

Hodges J., Kanemasu E. T., Tearg J. D., 1979. Modeling dry matter accumulation and yield of grain sorghum. Can. J. Plant. Sci., 59, 803-818.

Hodgkinson H. C., 1974. Influence of partial defoliation on photosynthesis, photorespiration and transpiration by lucerne leaves of different ages. Aust. J. Plant Physiol., 1, 561-578.

Kanemasu E. T., Hiebsch C. H., 1975. Net carbon dioxide exchange of wheat, sorghum and soybean. Canad. J. Bot. 53, 4, 382-389.

Kortschak H. P., Forbes A., 1969. The effects of shade and age on the photosynthesis rates of sugar-cane. Progr. photosynth. Res. 1, 383-387.

Kuroiwa S., 1970. Total photosynthesis of a foliage in relation to inclination of leaves. In * Prediction and measurement of photosynthetic productivity" Proc. IBP/PP technical Meet. Trebon 14-21 September 1969. Pudoc Wageningen, 79-89.

Kvet J., Ondok J. P., Necas J., Jarvis P. G., 1971. Methods of growth analysis In "Plant photosynthetic production - Manual of methods». Z. Sestak, J. Catsky, P. G. Jarvis Ed., Junk. The Hague, 343-384.

Lemaire Y., Robert G., 1973. Manuel du Planteur. Centre Technique de la Canne et du Sucre de Guadeloupe Ed., 200 pp.

Lemeur R., Blad B. L., 1974. A critical review of light models for estimating the shortwave radiation regime of plant canopies. Agric. Meteorol. 14, 255-286.

Ludlow M. M., Wilson G. L., 1971 a. Photosynthesis of tropical pasture plants. I-II luminance, carbon dioxide concentration leaf temperature, and leaf air vapour pressure difference. Aust. J. Biol. Sci., 24, 449-470.

Ludlow M. M., Wilson G. L., 1971 b. Photosynthesis of tropical pasture plants. III. Leaf Age. Aust. J. Biol. Sci., 24, 1077-1087.
McCree K. J., 1974. Equations for the rate of dark respiration of white clover and grain sorghum, as functions of dry weight, photosynthetic rate and temperature. Crop Sci., 14, 509-514.

More Herrero A., Bethenod O., Morot-Gaudry J.-F., 1980. Influence de la température sur les paramètres caractéristiques de la photosynthèse chez de jeunes plants de mais (Var. W64.A.). Physiol. vég. 18 (2), 301-312.

Nichiporovich A. A., 1967. Aims of research on the photosynthesis of plants as a factor of productivity. In "Photosynthesis of Productive Systems». Nichiporovich A. A. Ed., 3-36, Israel program for Sci. Translation Jerusalem.

Orgill J. F., Hollands K. G. T., 1977. Correlation equation for hourly diffuse radiation on a horizontal surface. Solar Energy, 19 , 357-359.

Penning de Vries F. W. T., 1972. Respiration and growth. In « Crop processes in controlled environment $"$ A. R. Rees, K. E. Cockshull, D. W. Hand, R. G. Hurd Ed., Academic Press. London, 327-347. Perrin de Brichambaut C., 1976. Météorologie et Energie : L'évaluation du "gisement" solaire. La Météorologie, 5 (6), 129-158.

Peters D. B., Cloucht B. F., Carves R. A., Stahl G. R., 1974. Measurement of dark respiration, evaporation and photosynthesis in field plots. Agron. J. 66, 460-462.

Robelin M., Mauget J. C., 1977. Comportement photosynthétique du noyer (Juglans regia L.). Premières observations sur un couvert continu obtenu à partir d'un semis haute densité. Ann. agron., 28 (6), 583-597.

Ryle G. J. A., Cobby J. M., Powell C. E., 1976. Synthetic and maintenance respiratory losses of $14 \mathrm{CO}_{2}$ in uniculm barley and maize. Ann. Bot., 40, 471-486.

Sinclair R., Thomas D. A., 1970. Optical properties of leaves of some species in arid South Australia. Aust. J. Biol. 18, 261-273.

Sinclair T. R., 1971. An evaluation of leaf angle effection maize photosynthesis and productivity. $\mathrm{PhD}$ Agriculture, plant culture, Cornell University.

Van Dillewijn C., 1954. Botanique de la canne à sucre. Traduction ed. C.T.C.S. Guadeloupe et Martinique, $391 \mathrm{pp}$.

Varlet-Grancher C., Bonhomme R., 1979. Application aux couverts végétaux des lois de rayonnements en milieu diffusant. II. Interception de l'énergie solaire par une culture. Ann. agron. 30 (1), 1-26.

Varlet-Grancher C., Bonhomme R., Chartier M., Artis P., 1981. Efficience de la conversion de l'énergie solaire par un couvert végétal. Oecol. Plant. (sous presse).

Varlet-Grancher C., Bonhomme R., Jacob C., Artis P., Chartier M. 1980. Caractérisation et évolution de la structure d'un couvert végétal de canne à sucre. Ann. agron. 31, 4, 429-454.

Waldron J. C., Glasziou K. T., Bull T. A., 1967. The physiology of sugar-cane. IX. Factors affecting photosynthesis and sugar storage. Aust. J. Biol. Sci., 20, 1043-1052.

Warren Wilson J., 1972. Control of crop processes. In « Crop processes in controlled environments". A. R. Rees, K. E. Cockshull, D. W. Hand, R. G. Hurd Ed., Academic Press, London, 7-30.

Wassink E.C., 1959. Efficiency of light energy conversion in plant growth. Plant Physiol. 34, 356-361.

Wit C. T. (de), 1965. Photosynthesis of leaf canopies. Versl. Landouwk. Onderz. 663, 1-57.

Wit C. T. (de) et al., 1978. Simulation of assimilation, respiration and transpiration of crops, Simulation Monographs. Pudoc Wageningen, $141 \mathrm{pp}$.

Woledge J., 1971. The effect of light intensity during growth on the subsequent rate of photosynthesis of leaves of tall fescue (Festuca arundinacea). Ann. Bot., 35, 311-322. 